\title{
LA ESTANCIA DE MARIA ZAMBRANO EN CHILE ${ }^{1}$
}

\author{
Maria Zambrano's stay in Chile \\ Antolín Sánchez Cuervo* \\ Sebastián Hernández Toledo ${ }^{* *}$
}

\section{RESUMEN}

Durante su estancia en Santiago de Chile entre 1936 y 1937, coincidiendo con los primeros meses de la guerra civil española, María Zambrano colaboró con diversos medios intelectuales favorables a la República española, publicó uno de sus primeros libros y varias antologías en la editorial Panorama, y escribió numerosos ensayos breves. El presente artículo plantea una aproximación a esta breve pero significativa etapa chilena de María Zambrano.

Palabras clave: María Zambrano, guerra civil española, compromiso intelectual, razón poética.

\begin{abstract}
During his stay in Santiago of Chile between 1936 and 1937, coinciding with the first months of the Spanish civil war, Maria Zambrano collaborated with diverse intellectual means favourable to the Spanish Republic, published one of his first books and several anthologies in publishing Panorama, and wrote numerous brief essays. The present article raises an approximation to this brief but significant this Chilean stage of Maria Zambrano.

Keywords: María Zambrano, Spanish civil war, intellectual commitment, poetic reason.

\footnotetext{
${ }^{1}$ La presente contribución ha sido realizada en el marco del proyecto de investigación El pensamiento del exilio español de 1939 y la construcción de una racionalidad política (FFI2012-30822), financiado por el Ministerio de Economía y Competitividad del Gobierno de España.

*Instituto de Filosofía-CSIC, Madrid, España. Correo electrónico: antolin.scuervo@cchs.csic.es

** Pontificia Universidad Católica, Santiago, Chile. Correo electrónico: srhernandez@uc.cl

Recibido el 16 de junio de 2013. Aceptado el 17 de octubre de 2013.
} 
Tras la crisis bursátil de 1929 y el desmoronamiento de la dictadura de Ibáñez (1927-1931), en Chile se produjo una fuerte crisis política, lo que mostró la injerencia de los militares en el gobierno y la imposibilidad de ejercer algún tipo de liderazgo sin un apoyo sólido de alguna clase social o de la elite política ${ }^{2}$. Posteriormente, entre 1932 y 1938, con el gobierno de Alessandri tuvo lugar la "restauración conservadora", la cual consistía en la vuelta a un sistema democrático de partidos donde el poder personalizado representaba orgánicamente a la sociedad. El control de los caudillismos y la garantía de un marco de legalidad política representativa que permitiera la creación de un consenso político en base a compromisos interclasistas dio así paso a una etapa reformista de larga duración, procurando constantemente los cambios y mejoras políticas y socioeconómicas de manera gradual para no desequilibrar la institucionalidad política ${ }^{3}$. En consecuencia, surge un nuevo orden social asociado a gobiernos de corte asistencialista, a pactos de estabilización nunca antes vistos en la política chilena y a alianzas políticas tales como el Frente Popular (1937-1941), favoreciendo la presencia de las necesidades obreras dentro de los sistemas burocráticos desde la segunda década del siglo XX.

En 1936, en medio de este contexto, los intelectuales chilenos y latinoamericanos centran su interés en Europa, especialmente en la Guerra Civil Española, manteniendo en un segundo orden las preocupaciones de la política nacional. Con esta inquietud nació ese mismo año Onda Corta, periódico que publicó siete números hasta marzo de 1937 con un papel difusor de ideas libertarias y del apoyo público por parte de intelectuales chilenos y latinoamericanos a los españoles republicanos. En este periódico destacaron las firmas de Enrique Espinoza, Augusto D’Halmar, Mariano Latorre, Ricardo A. Latcham, Marta Brunet, Mariano Picón-Salas, Manuel Rojas, José González Vera, Ezequiel Martínez Estrada, María Zambrano, entre otros; quienes manifestaron su apoyo a la libertad cultural y desaprobaron enérgicamente el fascismo y el imperialismo. Muchos de ellos formaban parte además de la sección local de la Liga para la Defensa de la Cultura constituida en París en 1936 por intelectuales de todo el mundo como expresión de rechazo de la política dictatorial y la censura cultural. El primer saludo de Onda Corta seńalaba en este sentido lo siguiente:

"Un grupo de trabajadores intelectuales ha constituido en Chile una Liga para defender la cultura, hoy perseguida en casi todo el haz de la tierra. La exaltación guerrera y

${ }^{2}$ Sobre esta cuestión, véase Maldonado, Carlos: Entre reacción civilista y constitucionalismo formal: las fuerzas armadas chilenas en el periodo 1931-1938. Chile: FLACSO, (1988): 14-16.

${ }^{3}$ Moulian, Tomás. Contradicciones del desarrollo político chileno, 1920-1990. Santiago de Chile: Ediciones LOM, (2009): 25. 
nacionalista, el imperialismo y el predominio del dinero, está ejerciendo en el mundo una opresión de la inteligencia y un estancamiento de la vida espiritual. Tanto la ciencia como la creación artística, en muchos países, están subordinadas a regímenes de fuerza que, fuera de entorpecer su libre desarrollo, pretenden hacerla servir los intereses materiales que ellos representan. La prueba de cuanto aquí se afirma está en la destrucción de libros, en la persecución racial y en la anulación de los derechos individuales incorporados a la vida civilizada" ${ }^{\text {. }}$

Este manifiesto logró establecer ciertos parámetros ideológicos seguidos por los intelectuales residentes en Chile, quienes, a propósito de la guerra española, dieron cuenta de una cultura política ecléctica que rondaba entre la izquierda y las ideas libertarias, haciendo de la libre difusión de la cultura sus principales postulados. De este modo, todos los colaboradores de Onda Corta se mostraron contrarios a todo tipo de represión y censura cultural, enfatizando por el contrario la legitimidad de la libertad de expresión. Sin embargo, a pesar de que el clima político mundial se encontraba muy activo a causa de la Guerra Civil Española y del surgimiento del fascismo a escala mundial, en el Chile de 1935 las ideas libertarias sólo fueron representadas - a nivel político- por la Federación Juvenil Libertaria, conformada por jóvenes del Partido Socialista que buscaban una propuesta ácrata. Esto los transformó en una de las orgánicas más importantes del anarquismo en los años 30, aunque su influencia y duración fue perecedera ${ }^{5}$.

Entre tanto, Onda Corta mostró ciertas tendencias libertarias que se impregnaron principalmente de ideas antifascistas, manifestando una amplia simpatía hacia los españoles republicanos. Buena muestra de ello era el siguiente fragmento, en el que se celebraba la aparición de El mono azul, revista editada por la Alianza de Intelectuales Antifascistas que había empezado a circular en Madrid en agosto de 1936:

"El mismo día que las tropas mercenarias del general Franco, armadas por Hitler y Mussolini, llegaban a las puertas de Madrid para estrellarse definitivamente contra el heroico pueblo español, después de prepararle durante más de cien días una "tenaza" militar irresistible [...] recibimos aquí en Santiago los primeros ejemplares del valiente periódico madrileño que se llama significativamente "El Mono Azul" [...]".

En efecto, no otra cosa que una victoria rotunda, significa la resistencia invencible que desde entonces ofrecen a los espadones de la tenaza los heroicos milicianos del mono azul... Porque urge decirlo, mono es el nombre que en la

${ }^{4}$ Manifiesto del Congreso Internacional de Escritores para la Defensa de la Cultura", Onda Corta, (Santiago de Chile), $\mathrm{n}^{\circ}$ 1, 15 de diciembre de 1936, p. 1.

${ }^{5}$ Del Solar, Felipe y Pérez, Andrés: Anarquistas. Presencia literaria en Chile. Santiago de Chile: Ril Editores, (2008): 53-54. 
maravillosa realidad de España, recibe el traje de los obreros industriales y azul, como en todas partes, su color, que los "nacionalistas" tińen de rojo, derramando a raudales su sangre generosa. Sangre del Pueblo! .

Este clima ideológico y de compromiso con la República Española se vio reforzado con la estancia en Chile de María Zambrano, colaboradora de Onda corta y Mono Azul, y discípula heterodoxa de Ortega y Gasset en el marco de la Escuela de Madrid, que para esos años ya se había distinguido por un desarrollo muy singular de la razón vital, así como por su implicación en el proyecto cultural republicano ${ }^{7}$. Había llegado a Valparaíso en noviembre de 1936 a bordo del "Santa Rita”, vía Balboa y Arica y apenas cuatro meses después del golpe militar del general Franco, acompañando a su marido, Alfonso Rodríguez Aldave, quien acababa de ser nombrado secretario de la Embajada Española ${ }^{8}$. Zambrano evocará su primer encuentro con América en su artículo "La tierra de Arauco", muy probablemente el primero de sus relatos de su salida de España en el contexto de la guerra y el exilio. En él abordaba una temática que será frecuente en numerosos escritos autobiográficos del exilio republicano español como la de la intimidad existente entre América y España -y muy especialmente la España derrotada-, así como su vocación universal y sus posibilidades aún inéditas frente a la violencia y el agotamiento de la cultura pragmática dominante. Esa intimidad se apreciaba en la experiencia común de la lengua, misma que, con palabras antiguas, hablaba en el pasado Cervantes y hablaba en el presente "el roto" o el Adán chileno; o en ciertas soledades cómplices como la de la misma España, retraída de Europa durante la modernidad y abandonada ahora, en plena guerra, a su propia suerte, y la del "roto" que deambula por las interminables avenidas y monótonas cuadrículas de Santiago.

La estancia de Zambrano en Chile duró apenas seis meses, los cuales fueron no obstante muy fecundos. Allí desarrolló numerosas actividades culturales

${ }^{6}$ Espinoza, Enrique: "Significación de 'El Mono Azul”, Onda Corta, Santiago, no 4, miércoles 6 de enero de 1937, p. 5.

${ }^{7}$ Véase el "Estudio introductorio" de Jesús Moreno Sanz a Zambrano, María: Horizonte del liberalismo. Edición de Jesús Moreno Sanz. Madrid: Morata, 1996; Bundgard, Ana: Un compromiso apasionado. María Zambrano: una intelectual al servicio del pueblo (1928-1939). Madrid: Trotta, 2009.

${ }^{8}$ Cf. la noticia de la llegada recogida en el diario La Estrella de Valparaíso el 18 de noviembre de 1936, de la que Pamela Soto García ofrece un fragmento en "María Zambrano en Chile", en María Zambrano. Ahora, ya, monográfico de la revista República de las letras (Madrid), no 89 (2005): 54.

${ }^{9}$ Revista de las Españas, no 102, Barcelona, junio 1938, pp. 21-22. Reeditado en Los intelectuales en el drama de España y escritos de la guerra civil. Presentación de Jesús Moreno Sanz. Madrid: Trotta, (1998): 222-227. Si bien no se publicó hasta 1938, es muy probable que este artículo se escribiera a finales de 1936 o comienzos de 1937 y que, debido a la guerra, su publicación se pospusiera hasta junio de 1938. Ciertamente, la Revista de las Españas, editada por la Unión Iberoamericana bajo la dirección de Corpus Barga, dejó de publicarse en 1936, pero en una noticia aparecida en La Vanguardia de Barcelona el 19 de mayo de 1938, se anunciaba su reaparición, con el no 101. Poco después, el 31 de julio, se anunciaba en el mismo periódico la publicación del no 102, en el que se incluía "La tierra de Arauco". La propia Zambrano figuraba entre los redactores de este número, junto al poeta Manuel Altolaguirre, entre otros. 
y propagandísticas a favor de la República española y se relacionó con diversos colectivos chilenos favorables a ésta última, tal y como ha mostrado Pamela Soto $^{10}$. Dictó conferencias, organizó conciertos, asistió a homenajes a la República española, colaboró con los periódicos Frente popular y Onda corta, escribió numerosos artículos, financió junto con su marido una antología poética del recientemente asesinado Federico García Lorca, y preparó un Romancero de la guerra. Además, publicó uno de sus primeros libros, Los intelectuales en el drama de España (Panorama, 1937) ${ }^{11}$, en el que, más allá de la cuestión española, planteaba una lúcida genealogía del fascismo en tanto que fenómeno arraigado en la racionalidad europea moderna. A su juicio - exponía en la primera parte del libroel fascismo hundía sus raíces en el idealismo, entendido este último como una construcción falsificadora de un saber absoluto o una inteligencia incondicional que explica y desarrolla, por sí misma y de manera autosuficiente, mediante categorías especulativas, la consistencia racional del mundo. Una inteligencia que por tanto soslaya y reprime aquellas realidades vitales no racionales, y sin embargo íntimas y radicales, cuyo estrangulamiento genera un estado de mala conciencia e impide una experiencia íntegra de la realidad. Su gran consecuencia no habría sido otra por tanto que el marchitamiento de la vida o una "sedimentación de sueños, deseos oscuros, desilusiones no formuladas, requerimientos incumplidos, que van aumentando" 12 hasta generar un resentimiento del que el fascismo, precisamente, será expresión brutal.

Bien distinto era el perfil del fascismo español, carente de antecedentes modernos. La ausencia en España de una cultura idealista ligada a la consolidación de una burguesía y al desarrollo de la lógica capitalista, motivó que el resentimiento fascista se nutriera en este caso de otras fuentes, algunas de ellas eficazmente manipuladas. Tales eran la conciencia de la decadencia de España durante los últimos siglos y su redescubrimiento en la estela de las generaciones del 98 y del 14, lo cual, mixtificado con elementos tradicionalistas y algunos tópicos importados del fascismo italiano, daba lugar a un nacionalismo falsificador y a medida de los sectores oligárquicos de la sociedad.

Sea como fuere, fascismos de unas y otras estirpes se habían concitado en la guerra de España y frente a ellos reivindicaba Zambrano, ya en la segunda

\footnotetext{
10 Cf. "María Zambrano en Chile”, Op. cit., 48-68.

${ }^{11}$ Fue reeditado en Madrid, Hispamerca, 1977; Barcelona, Anthropos, 1985 (junto con La tumba de Antígona y otros escritos, formando el volumen Senderos); Madrid, Trotta, 1998, Op. cit. En la "Presentación" a esta última (pp. 30-38), Jesús Moreno Sanz detalla los pormenores de estas reediciones.

${ }^{12}$ Zambrano, María: Los intelectuales en el drama de España y escritos de la guerra civil, ed. de 1998, p. 92.
} 
parte del libro, el compromiso del intelectual y la identificación de este último con el pueblo agredido, tal y como se venía plasmando en revistas emblemáticas como El mono azul, precisamente, y Hora de España, en la que colaboraban, entre otros, Dámaso Alonso, José Gaos, León Felipe, Rafael Alberti, Antonio Machado y José Moreno Villa. En estas revistas advertía Zambrano un rasgo que estará muy presente en sus escritos chilenos, como es la reivindicación de lo popular en la historia, la cultura y la lengua españolas, y su expresión trágica en el contexto de la guerra actual. Tal era, precisamente, el gran motivo de las dos antologías poéticas que publicó en Santiago, también en la editorial Panorama. Federico García Lorca. Antología (1937), había sido sufragada con el sueldo de diplomático de su marido ${ }^{13}$ y rendía homenaje al recientemente asesinado poeta andaluz. Iba precedida de los poemas "A Federico García Lorca” de Rafael Alberti y "El crimen fue en Granada" de Antonio Machado, así como de un breve ensayo de la propia Zambrano sobre la significación de su poesía, caracterizada sobre todo por su capacidad de expresar ese elemento popular en toda su autenticidad, sin la distancia del folklorista o de quien se limita a describir algo ajeno. Arraigada de manera íntima en lo más profundo de la sabiduría popular, la poesía de Lorca, de condición eminentemente dramática, daba así voz a la fuerza de la sangre, el presentimiento de la muerte y el trasiego entre la burla y la amargura. Con todo ello -proseguía Zambrano- Lorca elevaba la cultura popular a la condición de vanguardia artística y contribuía a rectificar el aislamiento del intelectual español y su separación de la sociedad, en el que se había visto sumido desde el siglo XVIII. Con Lorca y otros poetas como Rafael Alberti, Miguel Hernández y Arturo Serrano Plaja, pintoras como Maruja Mallo o músicos como Rodolfo y Ernesto Halfter, ese elemento popular renacía y la poesía dejaba de ser una cuestión de élite. Zambrano ubicaba esta nueva sensibilidad en torno a 1928, en los años inmediatamente anteriores al advenimiento republicano, haciéndolo, por eso mismo, posible.

La antología en cuestión comprendía una selección de los libros Canciones, Poema del cante jondo, Romancero gitano, Poeta en Nueva York y Llanto por Ignacio Sánchez Mejías, cerrándose el libro con la "Oda a Federico García Lorca" de Pablo Neruda, una breve biografía y un listado de obras suyas elaborados por la propia Zambrano.

La otra antología llevaba por título Romancero de la guerra española (1937) e iba precedida de un breve prólogo en el que Zambrano incidía de nuevo en la

${ }^{13}$ Así lo afirma la propia Zambrano en la "Introducción" a la edición facsímil de esta antología publicada por la Fundación María Zambrano en 1989. 
significación del elemento popular, en esta ocasión ligada sobre todo al lenguaje. El romance era presentado como la forma más antigua e incluso como la forma natural de la poesía española, en la que se habría recogido la historia más real de España, sumida no obstante en el olvido o confinada en ambientes marginales a partir del divorcio entre la poesía culta y la poesía popular consumado en el Renacimiento. Para Zambrano, la guerra actual, entendida como trance trágico del pueblo español, constituía un momento idóneo para la expresión de esta forma poética. La antología incluía así poemas, de calidad quizá un tanto desigual, de Antonio Machado, Rafael Alberti, Manuel Altolaguirre, José Bergamín, Vicente Aleixandre, Arturo Serrano Plaja, Pla y Beltrán, Luis de Tapia, Félix V. Ramos, Guillermo Lorente, Luis Pérez Infante, Antonio Aparicio, José Herrera Petere, Lorenzo Varela, Emilio Prados, Antonio Sánchez Barbudo, José Antonio Balbontín, Pedro Garfias, Mariano del Alcázar, Miguel Hernández, José María Quiroga Pla, Rosa Chacel, José Moreno Villa, Raúl González Muñón y Pablo Neruda.

Por otra parte, la antología de poetas chilenos Madre España, también editada por Panorama en 1937, incluía un breve texto de Zambrano a modo de epílogo, fechado en enero de 1937 bajo el título de "A los poetas chilenos de "Madre España". En unos términos que recordaban a algunos fragmentos de "La tierra de Arauco", apelaba a la "vida en potencia" y a las "infinitas reservas morales y sentimentales, humanas", del pueblo español, llamado en la hora trágica actual a forjar "un mundo nuevo [...] con la razón creadora y con la inteligencia activa en conjunción con esa sangre que corre a torrentes", bajo la síntesis fecunda "de dolor humano y razón activa, de la carne que sufre y la inteligencia que descubre". $\mathrm{Al}$ igual que en muchos otros escritos zambranianos breves de estos ańos, marcados por la guerra y el compromiso del intelectual, predominaba el tono propagandístico y militante, pero sin llegar a anular un cierto contenido reflexivo. En esta ocasión, "A los poetas chilenos de 'Madre España” constituye uno de los primeros textos en los que Zambrano emplea el término "razón poética", que usará posteriormente para identificar su particular síntesis de pensamiento y poesía. Es preciso más que nunca -afirmaba- "el ejercicio de la razón y de la razón poética que encuentra en un instantáneo descubrimiento lo que la inteligencia desgrana paso a paso en sus elementos. Es necesaria y más que nunca la poesía y por eso es que brota entre vosotros, hermanos chilenos que contribuís así a la lucha de España acompañándola, dándole vuestra voz de amor y esperanza [...]”. Esa voz era la de Vicente Huidobro, Carlos Préndez Saldias, Pablo de Rokha, Gerardo 
Seguel, Pablo Neruda, Winett de Rokha, Julio Barrenechea, Blanca Luz Brum, Volodia Teitelboim, Rosamel del Valle, Braulio Arenas, Hernán Cañas, Robinson Gaete, Julio Molina, Eduardo Anguita, Enrique Gómez, Juvencio Valle, Eduardo Molina, Helio Rodríguez y Carlos de Rokha, autores de la antología en cuestión.

Algunos de los textos de Zambrano que acompañaban a estas antologías se publicaron aparte en el diario Frente Popular, editado en Santiago y afín a la resistencia republicana en España. Tal fue el caso de "Los poetas chilenos de 'Madre España”" (22 de enero), "La vida de García Lorca” (12 de abril) y "Romancero de la guerra española” (3 de mayo). En este mismo diario, Zambrano publicó también por entonces "Conversación con María Zambrano" (14 de enero de 1937), "La Hora de España" (31 de marzo) y "La intelectualidad española y la República" (14 de abril), a los que hay que sumar dos artículos publicados en La mujer nueva con el título de "La mujer en la lucha española" (diciembre de 1936) y "Madrid" (mayo de 1937), así como otros dos que enviará desde España poco después de su regreso: "El II Congreso de los intelectuales" (Ercilla, agosto de 1937) y "La mujer en la lucha actual" (Frente Popular, 10 de septiembre de 1937). Todos estos artículos fueron reeditados por Pamela Soto García en 2005, como anexo a su ya citado trabajo ${ }^{14}$, y expresan en su conjunto las inquietudes y temáticas que venimos apuntando: la honda significación de lo popular y su interpretación desde un cierto nacionalismo cultural, su dimensión revolucionaria y su significación trágica actual bajo la violencia de la guerra y el fascismo, y su sentido interpelador hacia el intelectual; en el marco, todo ello, de una especie de filosofía de la historia de Espańa con referencias a su íntima relación con América.

El tono de los artículos de Zambrano publicados en Onda corta ${ }^{15}$ es más sobrio y reflexivo, de la misma manera que sus contenidos son algo más densos. Sin abandonar esa pequeña constelación temática anteriormente señalada, la amplían y al mismo tiempo la precisan. "La vocación de ser hombre" aborda de nuevo el compromiso del intelectual ante la guerra, aunque profundizando en esta ocasión en su sentido trágico y, al mismo tiempo, superador de contradicciones. "Unamuno y su contrario" vincula el pensamiento trágico del que fuera uno de los grandes maestros de María Zambrano con la circunstancia de la guerra, dejando entrever una discreta crítica a su ambigüedad ante la misma. Por lo demás, se trata

\footnotetext{
${ }^{14}$ Véase "III. Textos de María Zambrano. Chile. Cuba”, María Zambrano. Ahora, ya, Op. cit., 166-191.

${ }^{15}$ Se trata de tres artículos ausentes en las bibliografías de María Zambrano elaboradas hasta ahora, incluso en las más exhaustivas, que fueron recientemente encontrados por Sebastián Hernández Toledo. Concretamente, "La vocación de ser hombre" (no 1, 15 de diciembre de 1936, p. 4), "Unamuno y su contrario" (no 4, 6 de enero de 1937, p. 3) y “iMadrid, Madrid!" (no 6, 1 a quincena de marzo de 1937, p. 4).
} 
de uno de los primeros textos que Zambrano dedicará a Unamuno. “iMadrid, Madrid!", finalmente, conforma, junto con el artículo "Madrid" publicado en La mujer nueva, con otros artículos publicados en Espańa durante los años de la guerra y con algunos textos incluidos en su posterior ensayo autobiográfico Delirio y destino, una pequeña constelación de escritos sobre esta ciudad en la que confluyen una especie de sociología poética de sus marcas geográficas y realidades urbanas, y la evocación testimonial.

Todos los escritos anteriormente referidos conforman la que podría ser la obra chilena de María Zambrano, siempre con al reserva de que en el futuro puedan encontrarse algunos más. Su paso por Chile fue relativamente fugaz pero muy fecundo, y pudo ser más prolongado de no ser, precisamente, por el compromiso de Zambrano ante los acontecimientos que estaban sucediendo en España. Según dirá ella misma al cabo de los años, el embajador en Chile, Rodrigo Soriano, hizo lo posible para que su marido permaneciera allí cuando fue llamado a filas, declarando insustituible su labor diplomática, e incluso le ofreció a ella un cargo en la misma embajada, pero ambos expresaron su deseo de incorporarse a la lucha cuanto antes ${ }^{16}$. Tras un homenaje de despedida organizado por María Elena Caffarena y Jorge Jiles ${ }^{17}$, ambos partían hacia España el 11 de mayo de 1937, emprendiendo un viaje de regreso que Zambrano evocará en "Españoles fuera de España"18.

Si bien el impacto de la guerra civil en Chile -apunta Luis Vitale- fue pese a todo secundario ${ }^{19}$, a raíz de los constantes golpes de Estado de corte fascista en Latinoamérica y la victoria de Franco en España, Chile se convertiría, bajo el gobierno del Frente Popular, en un escenario propicio para acoger a exiliados de distintas naciones, nutriéndose de sus aportaciones en multitud de aspectos culturales, literarios, políticos y sociales. Tal fue el caso del exilio republicano español, que, ya sin Zambrano, retomará en 1939 el hilo de la historia que ella parecía haber comenzado. Durante la guerra civil espańola, el gobierno chileno se había visto envuelto en ciertas tensiones diplomáticas, tanto con el gobierno

\footnotetext{
${ }^{16}$ Véase la ya citada "Introducción” a Federico García Lorca. Antología, ed. facsímil de 1989.

${ }^{17}$ Caffarena era fundadora del Movimiento pro Emancipación de la Mujer en Chile, con la que Zambrano mantuvo una fluida relación, y Jíles era director de Frente Popular. Cf. Soto, Pamela, Op. cit.

${ }^{18}$ Hora de España (Valencia-Barcelona), VII, julio 1937, pp. 59-62.

${ }^{19}$ Vitale, Luis. Contribución a una historia del anarquismo en América Latina. Santiago de Chile: Ediciones Espíritu Libertario, (2002): 188.
} 
republicano como, después, con el gobierno de Franco ${ }^{20}$. Sin embargo, fructificó la iniciativa de Pedro Aguirre Cerda de ofrecer refugio a los republicanos que se encontraban exiliados en Francia, donde a cargo de Pablo Neruda se dispuso del buque carguero Winnipeg para recibir a más de 2.200 refugiados. La idea consistía en acoger sólo a obreros especializados que pudieran contribuir al desarrollo material del país, pero dentro de este colectivo también se encontraban numerosos militantes y políticos ligados a la izquierda y el anarquismo, lo cual repercutirá en el ámbito cultural, político y artístico del país.

En el plano intelectual, fue al cabo de una década que la guerra civil española volvió a tener alguna significación. Para entonces, el país estaba sumido en un sistema de partidos que había abandonado la acción a través de bloques políticos polarizados, como en 1936, gobernándose ahora mediante pactos y negociaciones que generaban una rotación ideológica diversa en los cargos ministeriales, con el denominador común de un Presidente perteneciente al Partido Radical ${ }^{21}$. Intelectuales de perfil libertario como Manuel Rojas, José Santos González Vera o Laín Diez, rememoraban entonces dicha guerra como "la única luz libertaria de esperanza”. Manuel Rojas escribía en este sentido lo siguiente:

“Desde 1917, año de la revolución rusa, no había ocurrido en el mundo un movimiento social que despertara tanta pasión como aquella guerra civil, y no cabe duda de que la guerra civil espańola fue, más que nada, un movimiento social. Desde que sonó el primer tiro y se enfrió el primer muerto, cada ser humano supo cuál era su causa y cuál su fila y nadie quedó sin la primera ni sin la segunda, excepto quizás los que no tienen ni la tercera ni la cuarta ni ninguna. Pero los que defienden al obispo y a su cepillo, al general y a su espadón, al banquero y a sus céntimos, al propietario y a su conventillo, al hacendado y a sus uñas, al negrero y su látigo [...] y los que defienden su propio negocio, esté ese negocio donde esté, en Roma o en Londres, en París o en Nueva York, en Tokio o en Buenos Aires o Santiago, todos supieron desde el primer momento de qué se trataba: de defender el negocio de todos y cada uno"22.

A modo de conclusión, podemos decir que la estancia de María Zambrano en Chile fue, pese a su brevedad, doblemente significativa. Por una parte, nos remite a un momento singular de su trayectoria vital e intelectual, caracterizado por el impacto de la guerra civil espańola. Este impacto activó sin duda un pensamiento

\footnotetext{
${ }^{20}$ Sobre esta cuestión, véase Nocera, Raffaele: Chile y la Guerra. 1933-1943. Santiago de Chile: Centro de Investigaciones Barros Arana, (2006): 35-42.

${ }^{21}$ Correa, Sofía et al. Historia del siglo XX chileno. Santiago de Chile: Editorial Sudamericana, (2001): 130.

${ }^{22}$ Rojas, Manuel. “Diez años”, Babel Revista de arte y crítica. Santiago, no 34 (julio-agosto 1946), pp. 24-25 Reeditado en Soria, Carmen (Comp.), Letras anarquistas. Articulos periodísticos y otros escritos inéditos. Santiago de Chile: Planeta, (2005): 196.
} 
militante y comprometido con la defensa de la legitimidad republicana en su tierra de origen, tan duramente agraviada en aquellos momentos. Pero no sólo eso. La dimensión internacional que pronto adquirió dicha guerra en medio de una Europa amenazada por el totalitarismo y a punto de estallar, ligada a la proyección universal del pensamiento de Zambrano, que durante la década de los treinta ya había dado sobradas muestras de originalidad, propició que sus expresiones chilenas no se redujeran a una dimensión meramente propagandística o reivindicativa -la cual no dejaría de ser, por lo demás, legítima y justificada-. En Los intelectuales en el drama de España, sobre todo, este mismo drama es el hilo conductor de una reflexión que se prolonga más allá de la cuestión española y que ofrece claves genealógicas de interpretación de la quiebra actual de la racionalidad europea, plasmada en el fenómeno del fascismo. Asimismo, su estancia en Chile constituía su primera experiencia americana, además de todo un antecedente del largo y casi interminable exilio que emprenderá sólo dos años después. Por primera vez, su reflexión sobre la cultura espańola, constante a lo largo de toda su obra, tomaba contacto con una realidad tan íntimamente ligada a la misma como la americana.

Por otra parte, dicha estancia nos remite también a una cuestión tan poco estudiada, relativamente al menos, como es la recepción en Chile de la guerra civil y el exilio españoles. Zambrano colaboró con revistas casi desconocidas como Onda corta, muy sensibles a dicha recepción, mientras que sus publicaciones y actividades fueron reseñadas en periódicos y revistas. Se relacionó vivamente con círculos feministas e intelectuales chilenos, así como con círculos literarios de los que surgió una antología de poemas dedicados a la "Madre España" que por entonces se desangraba. Y si bien no recalará de nuevo en tierras chilenas una vez consumada la derrota, su presencia también constituía todo un precedente del posterior exilio en esas tierras de otros muchos espańoles que se verán desposeídos de su ciudadanía republicana, algo que, más allá del célebre episodio del Winnipeg, apenas ha sido estudiado. En el ámbito intelectual y por citar sólo algunos ejemplos, llegarán a Chile dramaturgos como Ricardo Morales y pensadores como Ferrater Mora, Francisco Soler, Augusto Pescador o Cástor Narvarte. La estancia de María Zambrano en Chile constituye así la puerta de entrada a un laberinto pendiente aún de recorrer con detenimiento. 


\section{REFERENCIAS}

AA VV. "Manifiesto del Congreso Internacional de Escritores para la Defensa de la Cultura", Onda Corta (Santiago de Chile), no 1, 15 de diciembre de 1936, p. 1.

Bundgard, Ana. Un compromiso apasionado. María Zambrano: una intelectual al servicio del pueblo (1928-1939). Madrid: Trotta, 2009.

Correa, Sofía et al. Historia del siglo XX chileno. Santiago de Chile: Editorial Sudamericana, 2001.

Del Solar, Felipe y Pérez, Andrés. Anarquistas. Presencia literaria en Chile. Santiago de Chile: Ril Editores, 2008.

Espinoza, Enrique. "Significación de "El Mono Azul", Onda Corta (Santiago), no 4, miércoles 6 de enero de 1937.

Maldonado, Carlos. Entre reacción civilista y constitucionalismo formal: las fuerzas armadas chilenas en el periodo 1931-1938. Chile, FLACSO, 1988.

Moulian, Tomás. Contradicciones del desarrollo político chileno, 1920-1990. Santiago de Chile: LOM Ediciones, 2009.

Nocera, Raffaele. Chile y la Guerra. 1933-1943. Santiago de Chile: Centro de Investigaciones Barros Arana, 2006.

Rojas, Manuel. "Diez años", en Babel. Revista de arte y crítica (Santiago), no 34 (julio-agosto 1946), pp. 24-25.

Soria, Carmen (Comp.). Letras anarquistas. Artículos periodísticos y otros escritos inéditos. Santiago de Chile: Planeta, 2005.

Soto García, Pamela. "María Zambrano en Chile", María Zambrano. Ahora, ya, monográfico de la revista República de las letras (Madrid), no 89 (2005), pp.48-68.

Vitale, Luis. Contribución a una historia del anarquismo en América Latina. Santiago de Chile: Ediciones Espíritu Libertario, 2002. 
Zambrano, María. Horizonte del liberalismo. Madrid: Biblioteca Nueva, 1930. Reeditado por Jesús Moreno Sanz. Madrid: Morata, 1996.

. "La vocación de ser hombre", Onda Corta (Santiago de Chile) no 1, 15 de diciembre de 1936, p. 4.

. "Unamuno y su contrario", Onda Corta (Santiago de Chile), no 4, 6 de enero de 1937 , p. 3.

- “Madrid, Madrid!”, Onda Corta (Santiago de Chile), no 6, 1a quincena de marzo de 1937, p. 4.

Los intelectuales en el drama de España. Santiago de Chile: Panorama, 1937. Reeditado en Madrid: Hispamerca, 1977; Senderos. Barcelona: Anthropos, (1985): 11-200; Madrid: Trotta, 1998.

- Federico García Lorca. Antología (selección e introducción). Santiago de Chile: Panorama, 1937. Ed. facsimil en Vélez-Málaga, Fundación María Zambrano, 1989.

Romancero de la guerra española (selección e introducción). Santiago de Chile: Panorama, 1937.

. "Españoles fuera de España", Hora de España (Valencia-Barcelona), VII, julio 1937, pp. 59-62. Reeditado en Los intelectuales en el drama de España. Ensayos y notas (1936-1939). Madrid: Hispamerca, (1977): 180184; Senderos. Barcelona: Anthropos, (1985): 170-174; Los intelectuales en el drama de España y escritos de la guerra civil. Presentación de Jesús Moreno Sanz. Madrid: Trotta, (1998): 144-147.

-. "La tierra de Arauco", Revista de las Españas (Barcelona), no 102 (junio 1938), pp. 21-22. Reeditado en Los intelectuales en el drama de España y escritos de la guerra civil. Presentación de Jesús Moreno Sanz. Madrid: Trotta, 1998, pp. 222-227.

-- “Textos de María Zambrano. Chile. Cuba”, en María Zambrano. Ahora, ya, monográfico de la revista República de las letras (Madrid), no 89 (2005), pp. 166-191. 